\title{
Physics and Statistics of Medical Imaging
}

\author{
David G. Brown and Robert F. Wagner
}

\begin{abstract}
Extraordinary progress has been made over the last two decades in the development and dissemination of new medical imaging technologies. The development of computed tomography, positron emission tomography, and magnetic resonance imaging, as well as major innovations to the conventional imaging modalities, have revolutionized medical diagnostic imaging. Despite their many differences, all of these modalities can be viewed from a common perspective: being described in terms of the underlying physical properties imaged, the type of radiation/ detection system used to produce the images, and the imaging performance that the modality achieves. both in absolute terms and relative to that of a conceptual ideal observer.

This is a US government work. There are no restrictions on its use.
\end{abstract}

KEY WORDS: imaging system, noise, performance evaluation, radiation.

$\mathbf{M}$ ODERN DAY MEDICAL imaging dates from the radiograph in Fig 1. That radiograph taken by Roentgen in 1895 shows the skeletal structure of his wife's hand-and her wedding ring. Within a few years the use of $\mathrm{x}$-ray radiography with fluorescent intensifying screens became a standard part of clinical practice. ${ }^{1,2}$ Further advances were made with the gradual development of new $x$-ray methods, in particular, planar tomography and $\mathrm{x}$-ray-intensified fluoroscopy, and of radionuclide ${ }^{3}$ and ultrasound ${ }^{4}$ imaging techniques; however, plain film radiography remained by far the dominant imaging modality. A veritable explosion of new medical imaging technologies has occurred over the past two decades, with the invention of computed tomography $(\mathrm{CT}),{ }^{5}$ positron emission tomography (PET), ${ }^{6}$ magnetic resonance imaging (MRI), ${ }^{7}$ and such technological improvements as digital subtraction angiography (DSA). ${ }^{8}$ Exquisite images, such as the MRI study shown in Fig 2, are the result of this progress, progress that shows every evidence

From the Center for Devices and Radiological Health, Food and Drug Administration, Rockville, MD.

Address reprint requests to David $G$. Brown, Center for Devices and Radiological Health, FDA, 5600 Fishers Ln, Rockville, MD 20857.

This is a US government work. There are no restrictions on its use.

0897-1889/89/0204-0002\$00.00/0 of continuing in new areas, eg, superconducting quantum interference devices (SQUID) for the mapping of the miniscule magnetic fields generated by electrical currents in the human body. ${ }^{9}$

In spite of the obvious diversity of the many imaging systems making up the modern diagnostic armamentarium, they also share many common features. Each system images some physical parameter or combination of parameters describing some aspect of human anatomy or physiology. They each use sophisticated detection systems to interrogate a region of interest by means of some form of electromagnetic or acoustic radiation. Furthermore, as imaging systems, they are all noise limited and are therefore amenable to evaluation in terms of the whole framework of performance-evaluation measures developed in statistical detection theory and refined for use in evaluating medical imaging systems. Finally, for ease of analysis, they all may be viewed as consisting of two separate subsystems, one for gathering or detecting information about the patient, and one for displaying or coupling this information to the radiologist.

The concept of being noise limited was developed by Albert Rose in the 1940s for light photon imaging ${ }^{10}$ and was later extended by Dainty and Shaw. ${ }^{11,12}$ Rose illustrated this concept with the series of photographs shown in Fig 3. Each photograph in the series was made with about six times more light photons than the preceding one. As the number of photons increases, the noise in the image decreases, and the quality of the image improves, clearly showing the intimate connection among (1) the number of quanta in an image, (2) the level of noise in the image, and (3) the amount of information the image can convey. These observations lead naturally to the quantification of images in terms of the number of quanta they are worth and to the general formalism discussed below that is applicable to all imaging systems. This approach includes the use of signal-to-noise ratios and other descriptors that can be used as measures of efficiency relative to the performance of a perfect or ideal observer/ system, an imaging system that makes the best possible use of all of the information available to it in the interrogating radiation stream when 


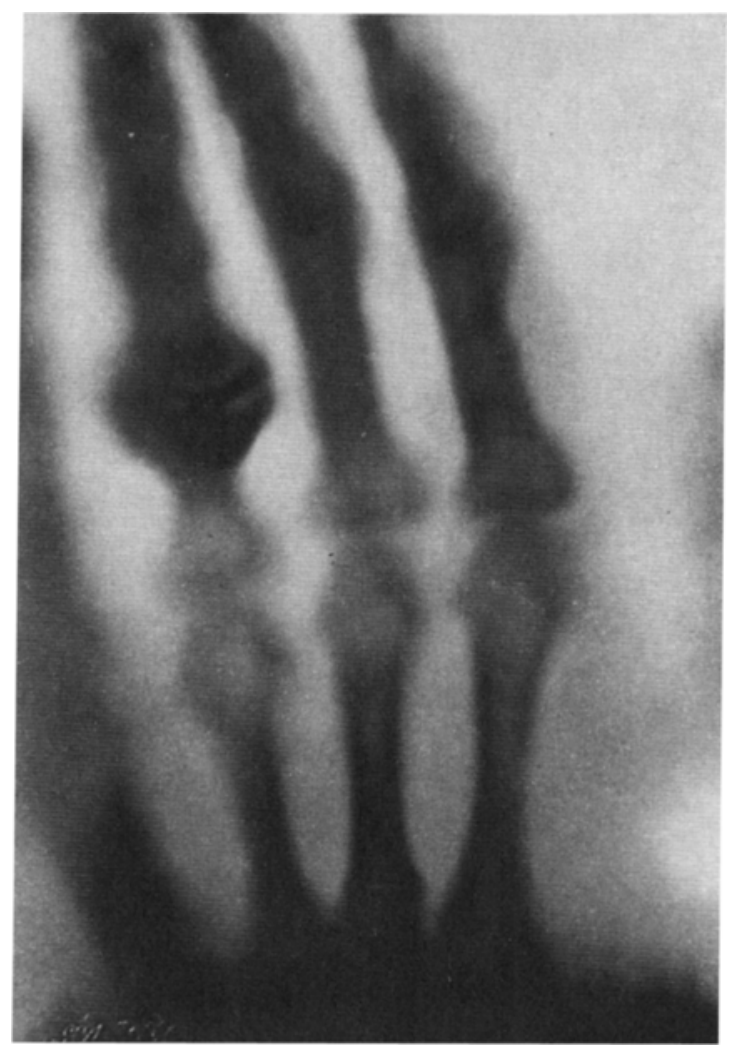

Fig 1. Early radiograph taken by Konrad Rontgen in 1895. It shows his wife's hand and her wedding ring. (Courtesy of Deutsches Museum, Munich, Federal Republic of Germany.)

making decisions about the structure of the underlying physical parameters of interest.

A second key concept required for a deeper understanding of medical imaging systems is the dichotomy between detection and display, as indicated in the simple block diagram of Fig $4 .^{13}$ Medical imaging systems are designed for (1) extracting information from the patient's body and (2) presenting it to a human observer for evaluation. These are very distinct processes, and an acceptable performance for one in no way guarantees that for the other. Much confusion was caused in the past by the failure to make this distinction-an understandable failure for plain film radiography in which the detection and display stages are so intimately related, but an obvious distinction for modern systems in which the computer memory commonly serves as a midway point between them. Fig 5 illustrates this point. Note how the severely underexposed radiograph in $5 \mathrm{~A}$ appears to be without diagnostic content. However, when it is suitably processed as is shown in 5B, it is discovered to actually contain a wealth of information that was previously inaccessible to the unaided human observer, but detected nonetheless. ${ }^{14}$

This report will explore further some of these ideas, beginning with a review of the kinds of physical parameters imaged and the forms of radiation and types of detection systems used to image them for various imaging systems. A discussion of the evaluation of imaging system performance will follow. We will not go into any great depth in explaining the physical principles behind the operation of any particular modality or the mathematical formalities supporting performance evaluation methodology, leaving these to the cited sources.

\section{PHYSICAL PARAMETERS}

The wide variety of physical parameters imaged by medical imaging systems is indicated in Table 1 . This great diversity of physical quantities available for imaging allows the patient to be viewed from many different perspectives. Thus, for example, bone-being high in average atomic number $(Z)$ and hence having high electron density-produces a strong signal in X-ray CT, but because it is low in free water and therefore supports relaxation times too short to be easily imaged, it produces only a weak signal in MRI. Similarly, nuclear medicine, using as its signal the radioactivity of chemically bound tracers, can give images as selective to tissue type or functional activity as the designer drugs that radiopharmacists can adapt to this task. For any physical parameter to be a candidate for medical imaging, all that is required is that it measurably vary over the body, and that this variation be of diagnostic relevance. In the language of imaging, its signal must be capable of standing sufficiently above the noise inherent in the imaging process, ie, its signal-to-noise ratio (SNR) must be sufficiently large for some clinically relevant variation in the parameter of interest to be observable. As seen in Table 1, many parameters have already been determined to be significant in this sense, and certainly many others are being and will continue to be examined in the future.

The actual image source situation is far more complex than is indicated in Table 1. For example, for plain film radiography there are not one 


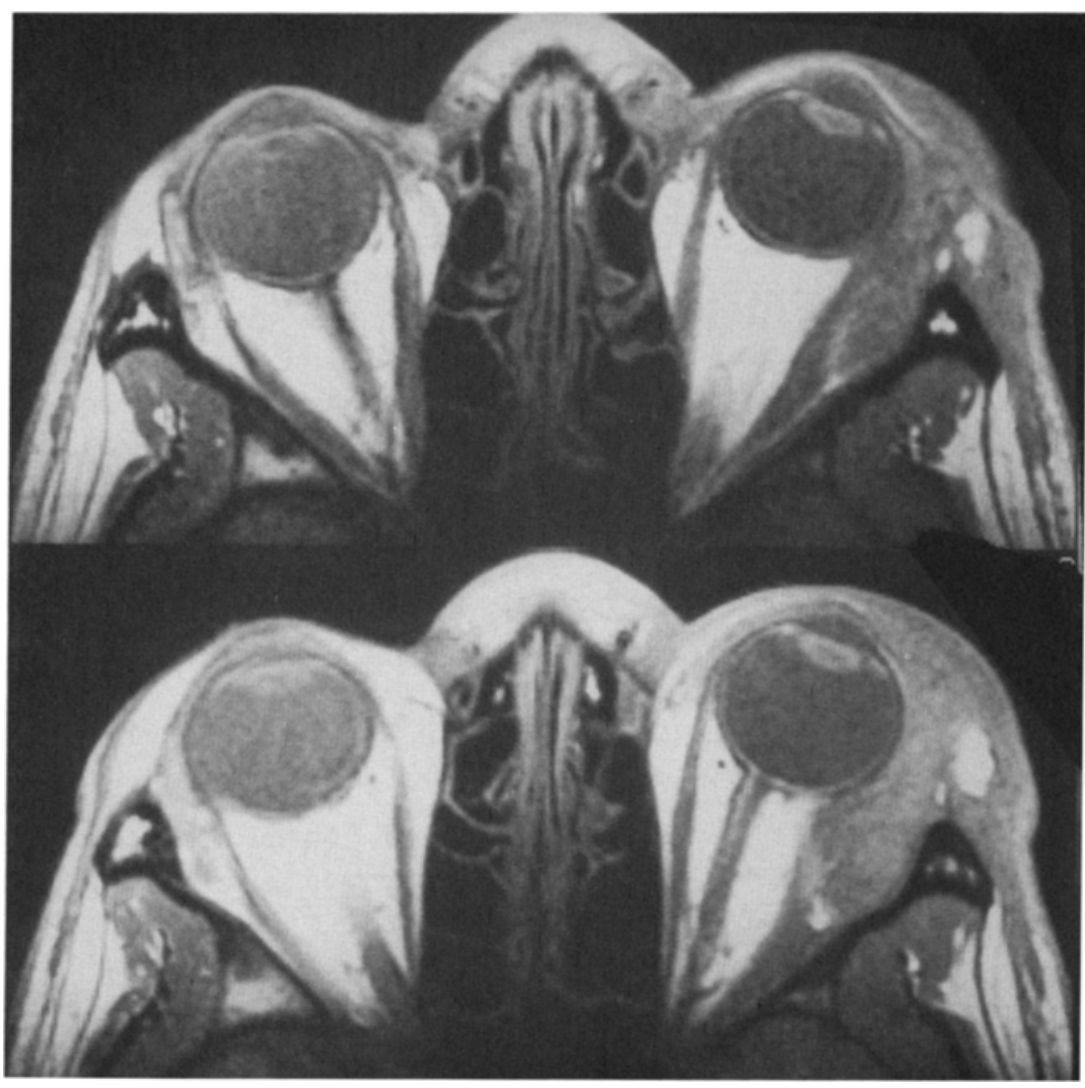

Fig 2. Magnetic resonance images. Two high resolution transverse slices on planes at the level of the patient's eyes (images obtained using surface coils). (Courtesy GE Medical Systems, Milwaukee, WI.) but several x-ray-interaction processes of interest. Alvarez ${ }^{15}$ notes that there are "twelve possible types of interaction processes," but that two of these dominate at typical diagnostic $x$-ray energies: Compton scattering ( $\mathrm{x}$-ray/quasi-free electron scattering) and the photoelectric effect ( $x$-ray absorption by a bound-electron atomic state). Because the dependence of these two processes on incident $\mathrm{x}$-ray energy is markedly different, radiographs taken at two different $\mathrm{x}$-ray energies can be used to generate fundamentally different images representing the two processes. Figure 6 shows examples of many tissue types that are separable based on that segregation into photoelectric versus Compton components. ${ }^{16}$

Figure 7 shows how this technique is used in practice to give separation between high- $Z$ (bone) and low-Z (soft-tissue) structures. ${ }^{17}$ This is particularly useful in chest radiography, as shown, in which there are many overlapping bone and soft-tissue features. In this way, such questions as the degree of tissue calcification may be resolved.
The versatility of nuclear medicine systems is even more pronounced. The parameter of interest is simply the specific activity of an administered radionuclide, and the art lies in somehow tagging a molecule, which will go to the site of interest within the body with an appropriate radionuclide. Very ingenious studies have been carried out based on this simple idea. In a way, music and speech themselves have been imaged in the work of Mazziotta and Phelps ${ }^{18}$ (Fig 8), in which cerebral glucose metabolism was imaged with a PET system while the subject listened to music or a work of literature, respectively. This study is a striking demonstration of the left-side/right-side brain functional dichotomy in operation. Thus, physiological function rather than merely morphology has become the subject of imaging.

Similarly, there are numerous parameters that may be imaged by MRI systems. Density of protons (or other nuclei with nonzero magnetic moment), relaxation times, (T1-relaxation mediated by the interaction of the nucleus with the entire surrounding lattice structure, and T2relaxation mediated by the interaction with spins 

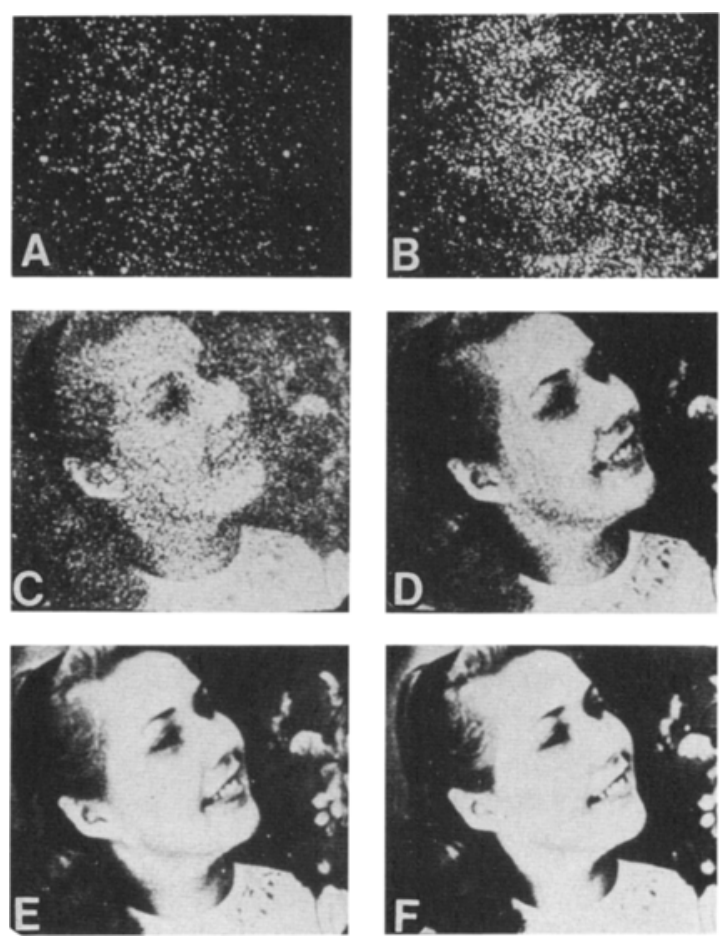

Fig 3. Series of pictures used by Albert Rose in evaluating the quantum efficiency of the eye. Pictures A-F were taken with $3 \times 10^{3}, 1.2 \times 10^{4}, 9.3 \times 10^{4}, 7.6 \times 10^{5}, 3.6 \times$ $10^{6}$, and $2.8 \times 10^{7}$ photons, respectively. (Reprinted with permission. ${ }^{10}$ )

of neighboring nuclei), and chemical shifts (changes in the resonant frequency caused by the influence of variations in the local magnetic environment) are those parameters most commonly enountered at this time. Because physiological processes often alter that environment, and MRI contrast agents are specifically designed to do so, MRI will increasingly share with nuclear medicine the ability to perform physiological imaging rather than simply providing the gross anatomical boundaries of organs. ${ }^{19}$

Note that there are inherent statistical as well as physical characteristics associated with each of the parameters measured. For nuclear medicine images, these are simply the statistics of the Poisson radioactive decay process. Even for radiographs, on a microscopic scale there are negligible statistical fluctuations associated with the variable number of atoms within any given measurement volume. However, for MRI imaging, this could possibly be a significant effect, at least for MRI microscopy, as outlined in Fig $9 .^{20}$

For further reading in these areas, good gen- eral texts such as those by Macovski ${ }^{21}$ and by Barrett and Swindell ${ }^{22}$ are suggested in addition to the references already cited.

\section{METHOD OF INTERROGATION}

How do we interrogate some portion of patient anatomy to accurately measure a given physical parameter of interest? By using what is probably a quite complicated (and expensive) piece of equipment to (1) subject it to some form of radiation and (2) detect the radiation (re)emitted from, transmitted through, or scattered by it. This section discusses the type of radiation used and the equipment that composes the imaging system. Of course, the physical parameter being imaged and the form of radiation used to image that parameter are very closely related. Typically, the radiation interaction cross sections are the parameters directly measured. However, it is a pedagogically appealing distinction and one that emphasizes other radiation concerns, eg, those of safety.

Table 2 gives a sampling of types of radiation and imaging modalities associated with each type. Acoustic radiation and many separate parts of the electromagnetic radiation spectrum are represented, so that it might be assumed that the various systems had little in common. However, two crucial radiation concerns are common to all medical imaging systems: the degree of attenuation of the radiation in the body and the potential for adverse health effects to the patient from the radiation.

All radiation carries energy, and too much energy deposited in the patient is harmful. Thus, through overheating if by no other mechanism, all forms of radiation are potentially dangerous. Of course, most forms of radiation are dangerous at much lower than thermal-heating levelshigh energy radiation ( $\mathrm{x}$ - and $\gamma$-rays) through ionization, and less energetic forms through cavitation (for acoustic radiation) or resonance

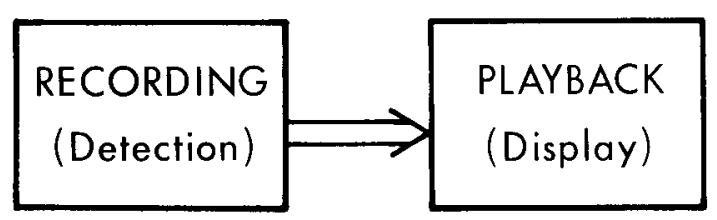

Fig 4. Simplest possible block diagram for assessment of imaging systems-showing division of a system into a recording or detection stage and a playback or display stage. ${ }^{13}$ 


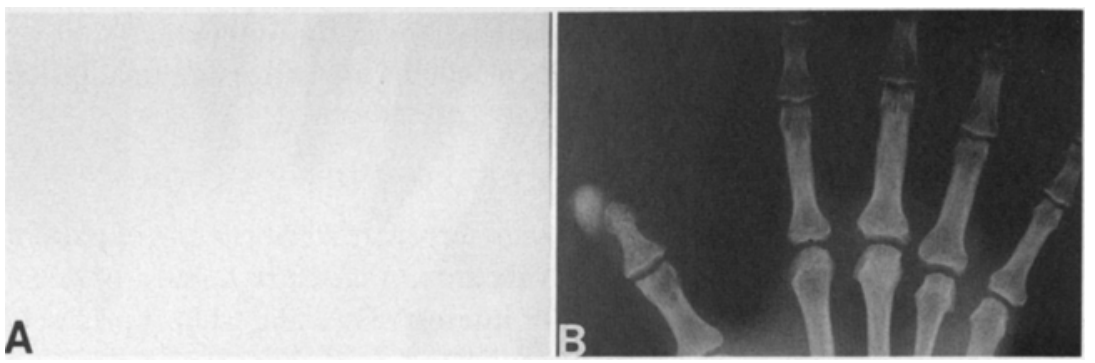

Fig 5. At left, severely underexposed radiograph showing faint outline of a hand. At right, radiograph after autoradiographic processing to enhance the image. (Process discussed in reference 14.)

effects. ${ }^{23,24}$ Therefore, historically, for every diagnostic imaging system there has been a concern with producing the best possible image for the least possible radiation dose to the patient, ie, radiation efficiency. This concern, and the time constraints on some modalities, eg, MRI, are the primary reasons for the noise limitations of medical imaging systems. Given enough radiation and/or enough time, noise could be reduced to a negligible level for most modalities. However, system noise, image artifacts, and the very clutter of the images themselves often provide a noise floor or radiation limit beyond which increases in radiation input provide no noticeable benefit in improved image quality. In practice, time and radiation constraints cannot be overcome, and radiation efficiency remains a critical requirement.

The relationship between patient radiation exposure and image quality has provided the inspiration for much of our own work at the Center for Devices and Radiological Health (CDRH). ${ }^{25,26} \mathrm{We}$ have been concerned with validating and encouraging efforts to increase the radiation efficiency of imaging systems for many years. In the early 1970s, for example, new phosphors became available from the television industry for possible use in $\mathrm{x}$-ray screens for screen-film radiography. In Fig 10, we illustrate the factor-of-two dose reduction attainable through use of screens constructed with the new phosphors to produce equivalent image quality. How this is accomplished and what we mean by

Table 1. Physical Parameters Imaged

\begin{tabular}{ll}
\hline \multicolumn{1}{c}{ Imaging System } & \multicolumn{1}{c}{ Physical Parameters } \\
\hline Radiography & Electron density \\
Nuclear medicine & Density of tracer molecules \\
Ultrasound & Density/compressibility changes \\
Magnetic resonance & Nuclear density, local magnetic \\
& environment \\
SQUID & Magnetic fields, microcurrents \\
\hline
\end{tabular}

equivalent image quality will be discussed in the following section.

Figure 11 illustrates the second point, that the degree of attenuation in the body is of critical importance. ${ }^{3}$ If the radiation attenuation is too great, patient exposure will reach intolerable levels before enough radiation manages to escape from the patient to allow sufficiently precise measurements by the detectors. On the other hand, if the attenuation is very small, once again too great a level of radiation may be required, in this case because the contrast (the difference in the data for regions that differ in the parameter of interest) will otherwise be too small to give a useful image. Figure 11 describes the case of the electromagnetic spectrum; however, a similar condition holds for the acoustic frequency spectrum, with attenuation increasing dramatically

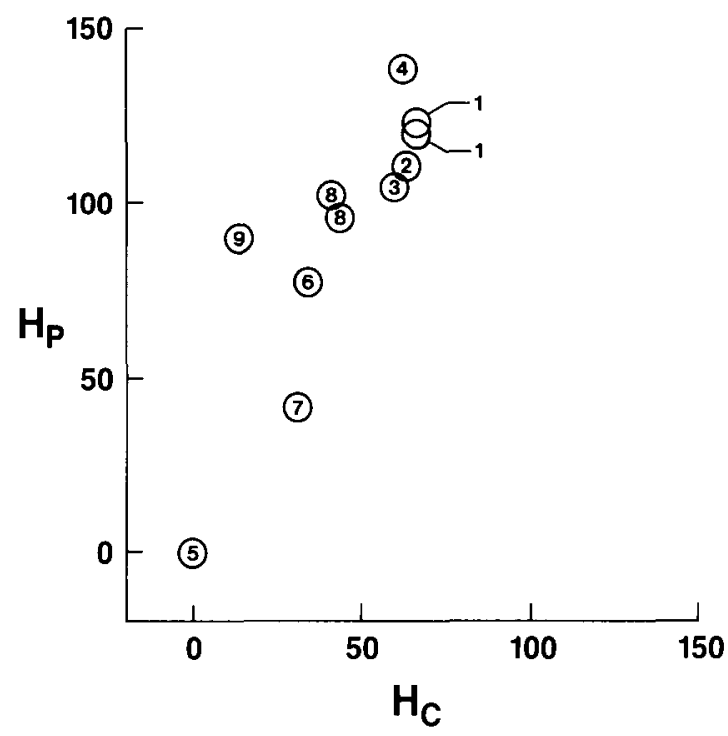

Fig 6. Plot of the photoelectric $\left\langle H_{p}\right\rangle$ and Compton scattering $\left(H_{c}\right)$ components for nine different body materials, showing their separability in this space. Values are scaled using generalized Hounsfield units. Materials: 1 , clotted blood; 2, muscle; 3, liver; 4, pancreas; 5, water; 6 , gray matter; 7, white matter; 8, meningioma; 9, neuroma. (Reprinted with permission. ${ }^{16}$ ) 

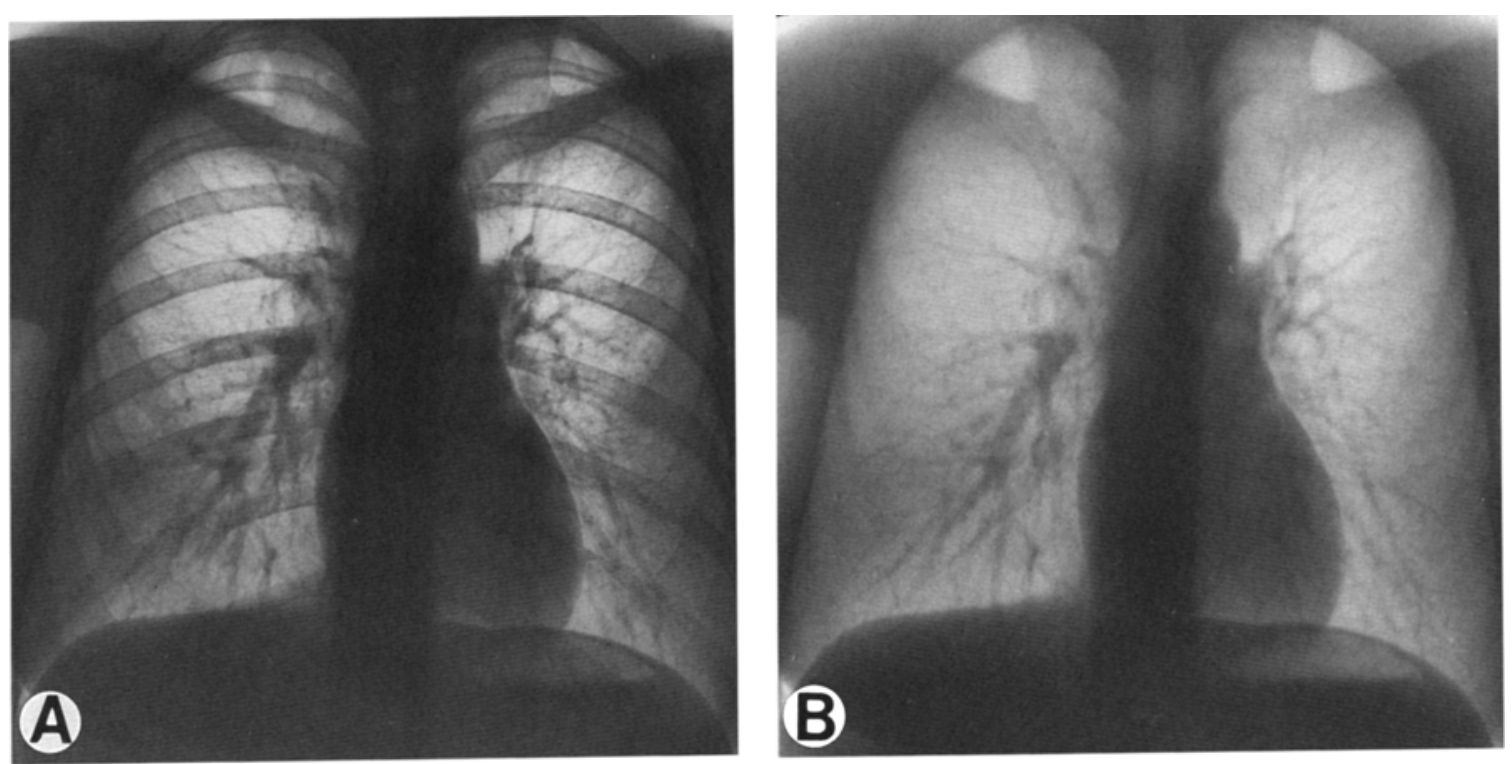

Fig 7. Dual-energy chest radiography images. (A) Normal radiograph, (B) soft tissue image, (C) bone image. (Courtesy G.T. Barnes, University of Alabama at Birmingham.)

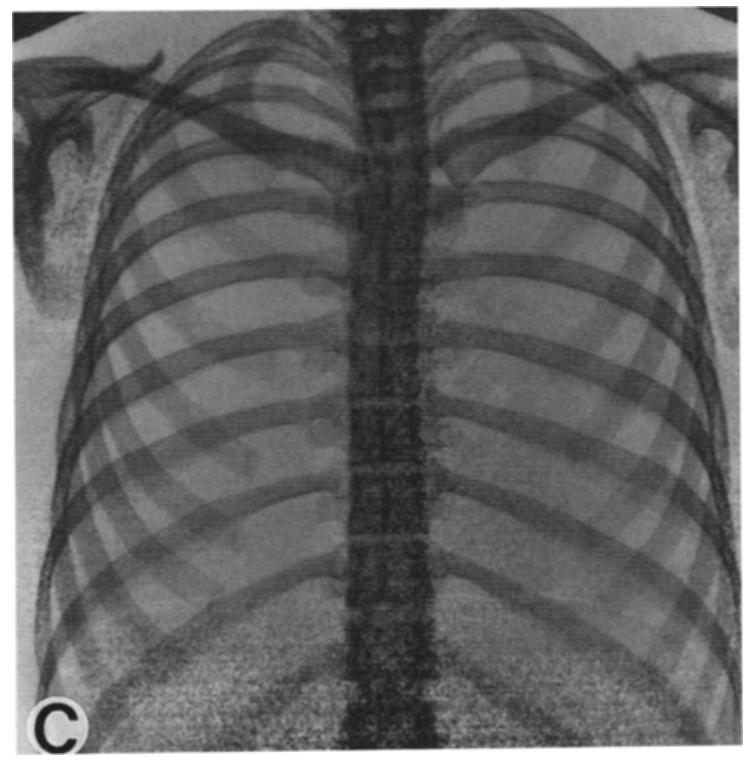

at higher frequencies. ${ }^{4}$ Note that the two regions with suitable intermediate attenuation correspond to the $\mathrm{x}$-ray spectrum of diagnostic radiology and the radio frequency region used by MRI and some prototype microwave imaging systems. ${ }^{27}$

Another concern relative to the interrogating radiation is the resolution constraint that may be imposed by the wavelength of the radiation. For example, one of the difficulties faced by microwave imaging is that the relatively long wavelengths that it uses preclude obtaining the degree of resolution of competing imaging modalities. Fortunately, this limitation does not apply to

modalities such as MRI, in which localization is achieved by other means.

Finally, there are obvious technical considerations affecting the use of any form of radiation. In particular, the state of the art in radiation detectors has played a key role in the feasibility of many imaging modalities. This is true not only for the reliability and quality of such detectors but for their size as well, which is directly related to the resolution capabilities of the systems. In general, detection systems for very low-energy (radio frequency) and very high-energy (particle) radiation are especially demanding techno- 


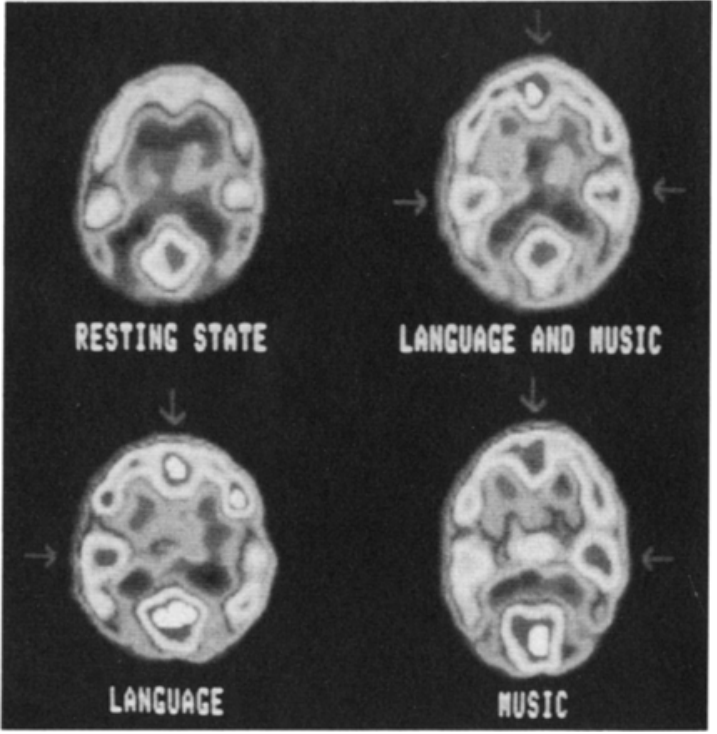

Fig 8. Cerebral glucose metabolism measured with PET system using ${ }^{18}$ F-lebeled fluorodeoxyglucose. Auditory system stimulation with language (Sherlock Holmes story) and music (Brandenburg Concertos). (Courtesy M.E. Phelps, University of California, Los Angeles.)

logically; eg, high-energy $\gamma$ rays are difficult to localize accurately.

This leads directly to consideration of the overall imaging system or imaging chain for a particular modality. As an example, Table 3 lists the major components of the imaging chain for the case of plain film radiography, but a similar schematization could be given for any imaging
Table 2. Radiation Used by Imaging System

\begin{tabular}{ll}
\hline \multicolumn{1}{c}{ Imaging System } & \multicolumn{1}{c}{ Radiation } \\
\hline Radiography & X-rays \\
Nuclear medicine & $\begin{array}{l}\text { Gamma rays } \\
\text { (from internal emitters) }\end{array}$ \\
Ultrasound & Acoustic \\
MRI/SQUID & Radiofrequency \\
Thermography & Infrared \\
$\quad$ (thermal mapping of body) & \\
Diaphanography & Optical \\
$\quad$ (transillumination using light) & \\
\hline
\end{tabular}

system. The elements of a system can generally be divided into those that occur before or those that occur after radiation is introduced into the body, ie, those elements either on the source side or the detector side of the patient. For the $\mathrm{x}$-ray case, the source side contains the $\mathrm{x}$-ray generator/ tube itself and the various filters and apertures used to control the incident radiation (energy spectrum and direction/location of incidence). The detector side contains the means to discriminate against unwanted scattered radiation (grid) and the actual radiation detector/recording device. The imaging chains for the various imaging systems are composed of similar kinds of components, each with its own function and limitations and its own potential for degrading system performance.

In our laboratory, we have examined the components of the $\mathrm{x}$-ray imaging chain in considerable detail. We have verified and encouraged

Energy levels split in a magnetic field $\mathrm{H}$

(spin up/spin down)

$$
\begin{aligned}
& \Delta \mathrm{E}=\mathrm{h}(\gamma / 2 \pi) \mathrm{H} \\
& \mathrm{h} \text { is Planck's constant } \\
& \gamma \text { is the gyromagnetic ratio } \\
& (\gamma / 2 \pi=42.6 \mathrm{MHz} / \text { tesla for protons })
\end{aligned}
$$

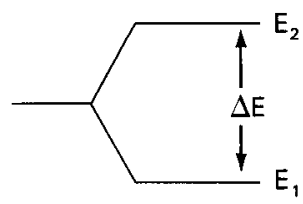

This leads to an excess population in the lower energy state $\left(\mathrm{N}_{1}\right)$ versus the population in the upper state $\left(\mathrm{N}_{2}\right)$ according to the Boltzmann factor at a given temperature $T$,

$$
\begin{aligned}
N_{1} / N_{2} & =\exp (\Delta E / k T) \\
& \simeq 1.00000696
\end{aligned}
$$

(for $\mathrm{H}=1$ tesla, $\mathrm{kT}=1 / 40 \mathrm{eV}$ )

Given a sample volume $V_{\mathrm{s}}$ we can use the binomial probability distribution to estimate the relative "noise" $(\sigma / \mu)$ caused by the fluctuations in the excess of protons in the low versus high energy states:
$V_{\mathrm{s}} \Rightarrow$
$1 \mathrm{~cm}^{3}$
$\sigma / \mu \Rightarrow$
$1.2 \times 10^{-6}$
$1 \mathrm{~mm}^{3}$
$(.01 \mathrm{~mm})^{3}$
$3.8 \times 10^{-2}$

Fig 9. Statistics of the magnetic resonance measurement process. Note that noise levels don't reach the $1 \%$ leveltypical of thermal noise-until dimensions on the order of .01 mm. 


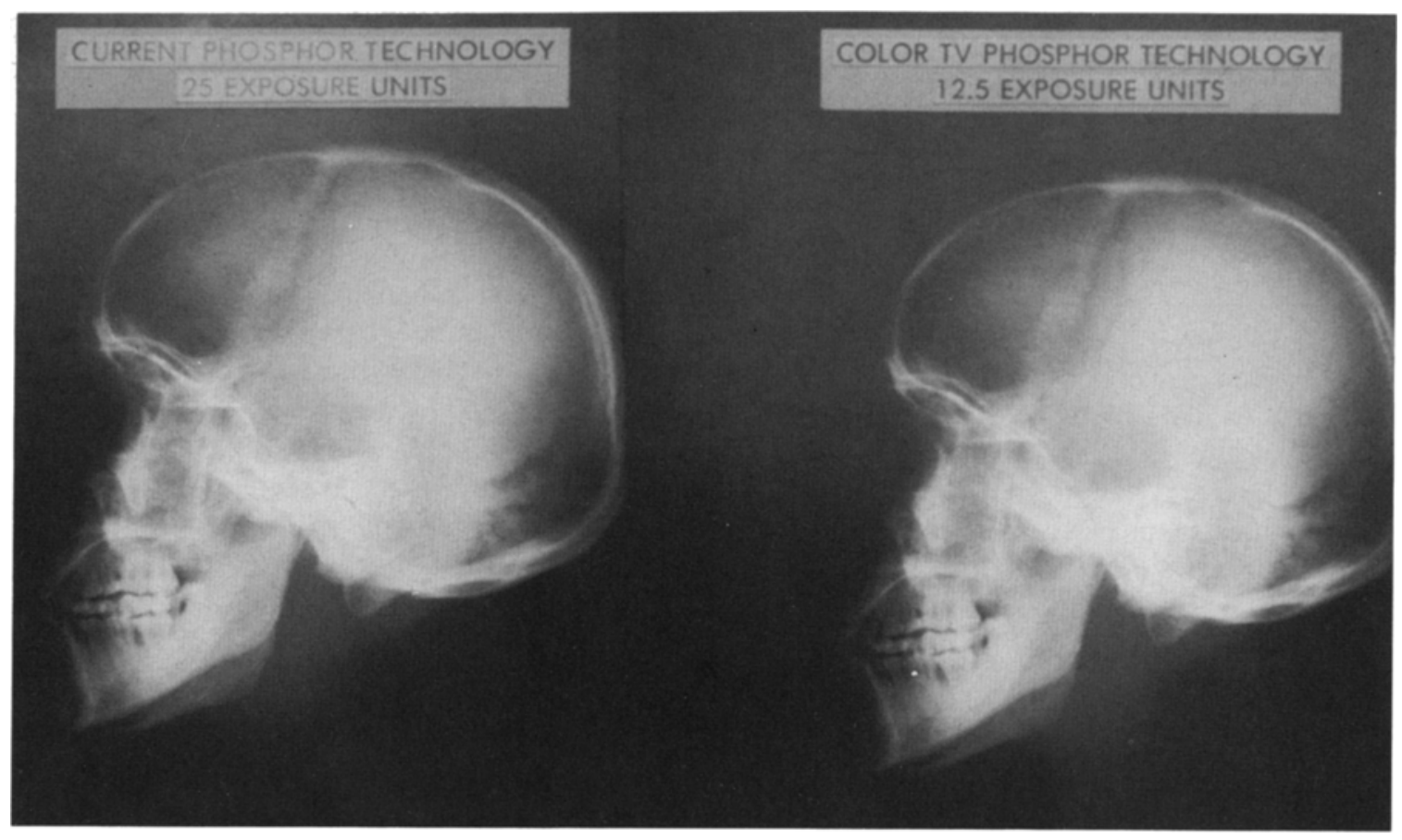

Fig 10. Skull radiographs showing factor-of-two dose savings (at left) for the new-technology (rare earth phosphor) screens versus the older (calcium tungstate) screens.

implementation of improvements in dose efficiency through system optimization that could lead to a degree of dose reduction of four or greater for some common procedures. ${ }^{28,29} \mathrm{An}$ other example of the kind of imaging system dose inefficiency that has concerned us is given in Fig 12. The width of the dose profile for a CT scan from one particular system is shown to be considerably greater than that of the imaged volume, resulting in wasted radiation which represents a very sizeable portion of the radiation exposure to the patient. ${ }^{30}$

One additional facilitating factor vital to the success of modern medical imaging systems should not pass unnoticed-the computer. Without the computational power provided by the modern computer, none of the imaging systems developed over the last two decades would have

Fig 11. Transmission fraction of electromagnetic radiation through $25 \mathrm{~cm}$ of soft tissue as a function of wavelength. (Albert Macovski, Medical/maging Systems, 1983, p 3. Reprinted by permission of Prentice Hall, Inc, Englewood Cliffs. NJ.)

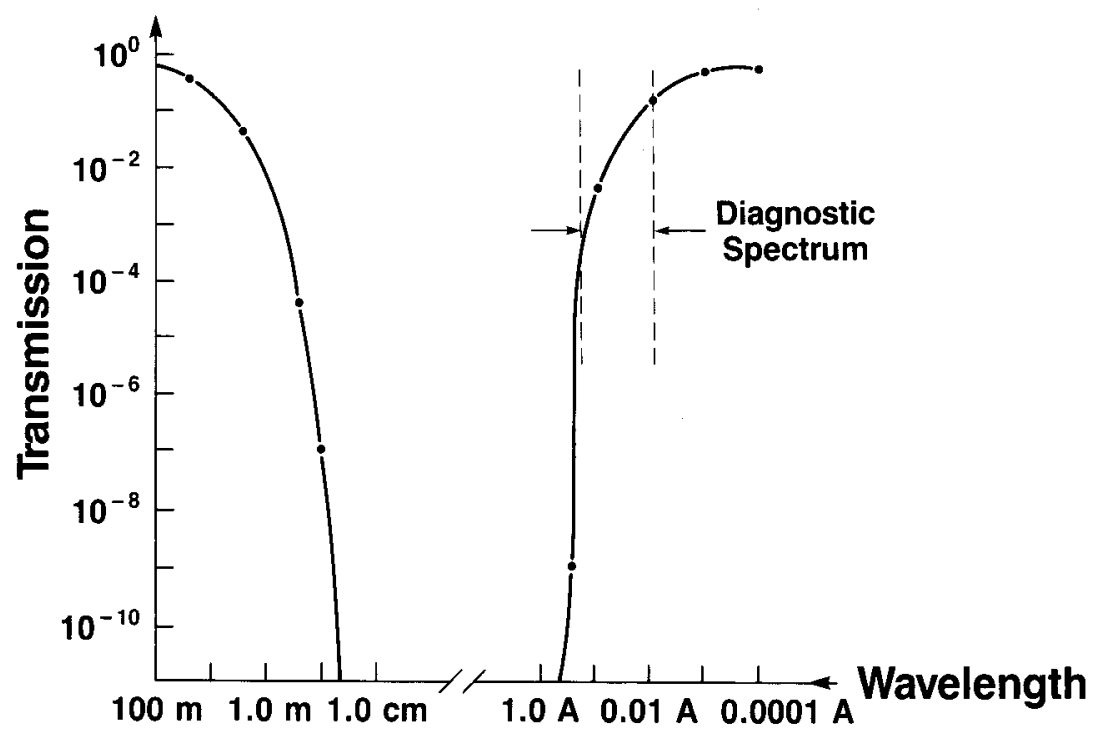


Table 3. Radiography Imaging Chain

\begin{tabular}{cl}
\hline \multicolumn{1}{c}{ Source Side } & Detector Side \\
\hline High-voltage generator & Table top \\
Tube & Grid \\
focal spot & Cassette \\
window & Screen \\
Collimator & Film \\
Filtration & Film processing \\
\hline
\end{tabular}

been feasible. ${ }^{31}$ These new systems reconstruct images of sections through patient anatomy from vast amounts of data that otherwise could not be directly interpreted by a human observer. The mathematical complexity of these systems' operations requires the very high level of computing speed and vast amounts of memory available with modern computers.

\section{PERFORMANCE EVALUATION}

This report has described how any imaging system may be characterized by the physical parameter(s) it images and the technical means it uses to form these images. It is also characterized by its imaging performance-how good a job does it do? This question may be answered in two ways: (1) by a quantitative description of the images themselves, and (2) as an efficiency in comparison with the performance of an "ideal" imaging system, ie, one with access to the same stream of information, carried by emitted or transmitted radiation, but with perfect collimators, detectors, and other system compo- nents. ${ }^{12,20-22}$ These approaches will be reviewed, primarily for the detection stage of the system, but with a brief discussion of the display stage as well.

\section{Detection Stage}

The resolution and noise properties of an image are the two major concerns at the detection stage of an imaging system, as distinct from such display-stage parameters as contrast scale and windowing. In any practical imaging system the images of (sufficiently) small objects appear smeared out or blurry to some extent, or to use mathematical terminology, the values of neighboring picture elements are correlated. Similarly, for noise-limited systems, images of even a completely homogeneous object have a blotchy or grainy appearance. Degraded resolution and system noise are the twin resident demons of all medical imaging systems. They arise naturally from the finite apertures and limited radiation exposures of real systems, and their quantification is the primary task of image assessment. Further defects, such as image nonuniformity and other image artifacts, may be very important; however, they tend to be more directly dependent on the type of imaging modality, and will not be discussed further here. ${ }^{13}$

Two simple measures of resolution and noise come readily to mind. For resolution, the image of a "point" source, ie, a source small in comparison with the apertures of the system, referred to

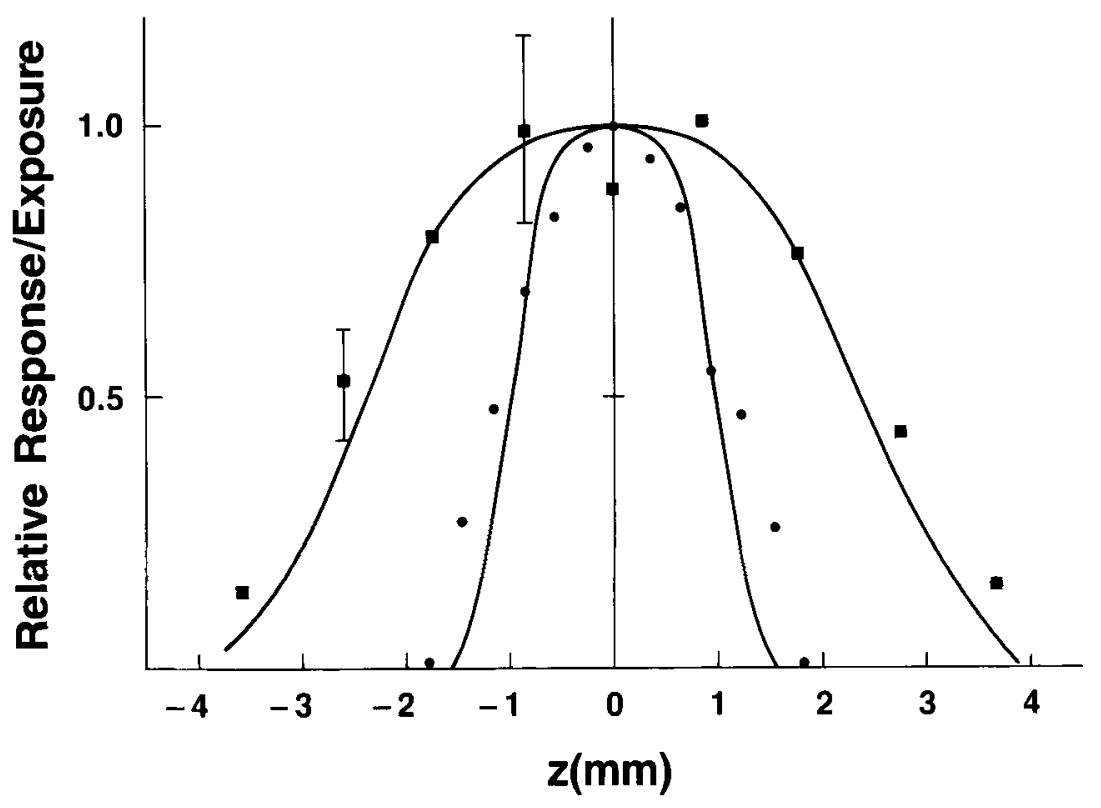

Fig 12. Relative dose louter curve) and imaging sensitivity (inner curvel 88 a function of distance (z) perpendicular to the image plane. Note how for this particular CT system most of the radiation passes outside of the image volume and does not contribute to the image. ${ }^{30}$ 
as the point spread function (psf), is the obvious choice. ${ }^{33}$ Its full width at half maximum (FWHM) frequently has been used to characterize system resolution. This is a great oversimplification, especially when the tails of the distribution are significant; however, it provides a single parameter for rough comparisons among similar systems. For noise, the corresponding single parameter is the standard deviation. The standard deviation (or its square, the variance, $\sigma^{2}$ ) is calculated from an image of a homogeneous source and for a given picture element (pixel) or measuring aperture size. This is unfortunately even less satisfactory for noise than FWHM is for resolution, because the correlation of neighboring pixels mixes together the noise and resolution properties, making $\sigma^{2}$ dependent on aperture size. It is useful, however, as a quality-control check on the variation over time of any particular imaging system. Moreover, it can be extended to be a more complete descriptor, the autocorrelation $(C(\underline{\Delta x}))$ of the noise:

$$
\mathrm{C}(\underline{\Delta \mathrm{x}})=\langle\Delta \mathrm{I}(\underline{\mathrm{x}}+\underline{\Delta \mathrm{x}}) \Delta \mathrm{I}(\underline{\mathrm{x}})\rangle
$$

where $\Delta I$ is the noisy image signal (I) minus the mean signal $(\langle\mathrm{I}\rangle)$, and the underline indicates a vector quantity, eg, $\underline{x}=(x, y)$ for the twodimensional image case. Note that $C(\underline{\Delta x})$ reduces to $\sigma^{2}$ when

$$
\underline{\Delta x}=0 .
$$

To obtain measures with greater theoretical significance and ease of interpretation, we must find a set of coordinates where values in adjoining locations or channels are uncorrelated, unlike pixels in the image signal. At least for lowcontrast signals (small relative signal differences) this is accomplished for many types of imaging systems by going from image space $(x, y)$ to spatial frequency coordinates $\left(f_{x}, f_{y}\right)$, using the Fourier transformation:

$\mathrm{I}(\underline{\mathrm{f}})=\int \mathrm{I}(\underline{\mathrm{x}})[\cos (2 \pi \underline{\mathrm{f}} \cdot \underline{\mathrm{x}})+\mathrm{i} \cdot \sin (2 \pi \underline{\mathrm{f}} \cdot \underline{\mathrm{x}})] \mathrm{d} \underline{\mathrm{x}}$ where $\mathrm{I}(\cdot)$ is the image in the respective space. The meaning of this operation is illustrated in Fig 13, where the real (cosine) part is shown for a given angular frequency $\omega=2 \pi|\mathrm{f}|$ and direction $\theta=$ arctangent $\left(\mathrm{f}_{\mathrm{y}} / \mathrm{f}_{\mathrm{x}}\right){ }^{34}$ The real part of $\mathrm{I}(\omega, \theta)$ is obtained by "beating" the cosine wave of frequency $\omega$ and direction $\theta$ against $\mathrm{I}(\underline{\mathrm{x}})$.

In this new spatial-frequency coordinate sys-

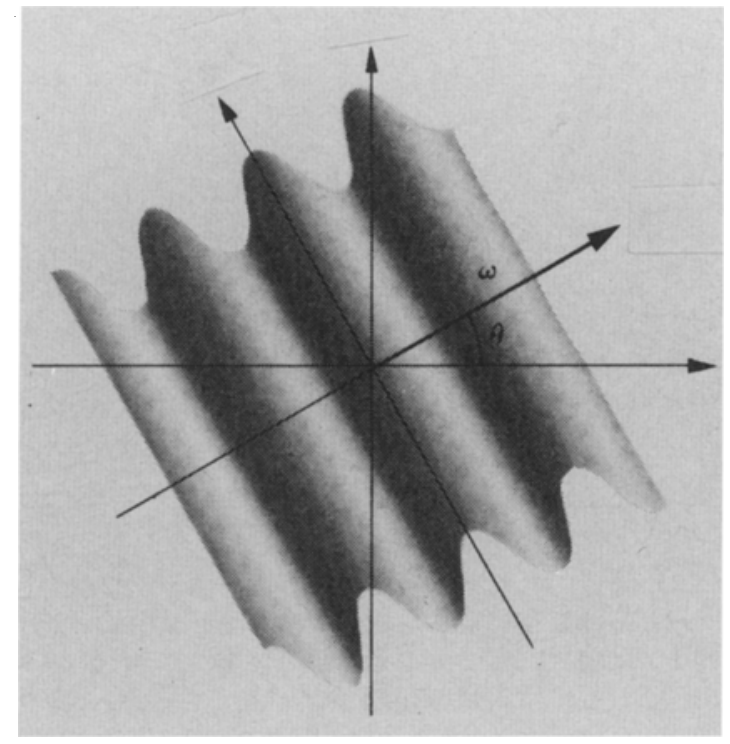

Fig 13. Pictorial representation of the real (cosine) factor of the Fourier integral to determine the Fourier component at spatial frequency $f=\omega / 2 \pi$ and direction $\theta$ with respect to the abscissa. $1 \mathrm{O}^{\prime} \mathrm{Neil}$ EL. Introduction to Statistical Optics, @ 1963, Addison-Wesley Publishing Co, Reading. MA. Page 23. Reprinted with permission.)

tem our measures of resolution and noise are simply the Fourier transforms of the psf and noise autocorrelation functions, and are referred to as the modulation transfer function (MTF) and noise-power spectrum (NPS), respectively. Figures 14 and 15 show examples of MTF and NPS curves from interlaboratory comparisons on screen-film radiography systems. ${ }^{35-37}$ Over the last 15 years these measurements have become standardized, and major medical equipment and medical-film manufacturing companies have begun making them on their systems and releasing them to their customers. They are useful because they indicate the level of resolution and noise expected in a system for finer and finer signal structure (higher and higher spatial frequency). For example, note that for the par-speed/XRP screen-film system, structure on the scale of 1 $\mathrm{mm}$ (spatial frequency 1 cycle per millimeter $\left.\left[\mathrm{mm}^{-1}\right]\right)$ is transmitted through the system at about five times the amplitude of $1 / 5-\mathrm{mm}$ (5 cycles per millimeter) structure and more than 10 times that of $1 / 10-\mathrm{mm}$ structure.

Why bother with this kind of complication when describing noise? Figure 16 provides an example of why this approach is necessary when considering noise properties. ${ }^{38}$ Both images in Fig 16 have the same total noise variance; how- 


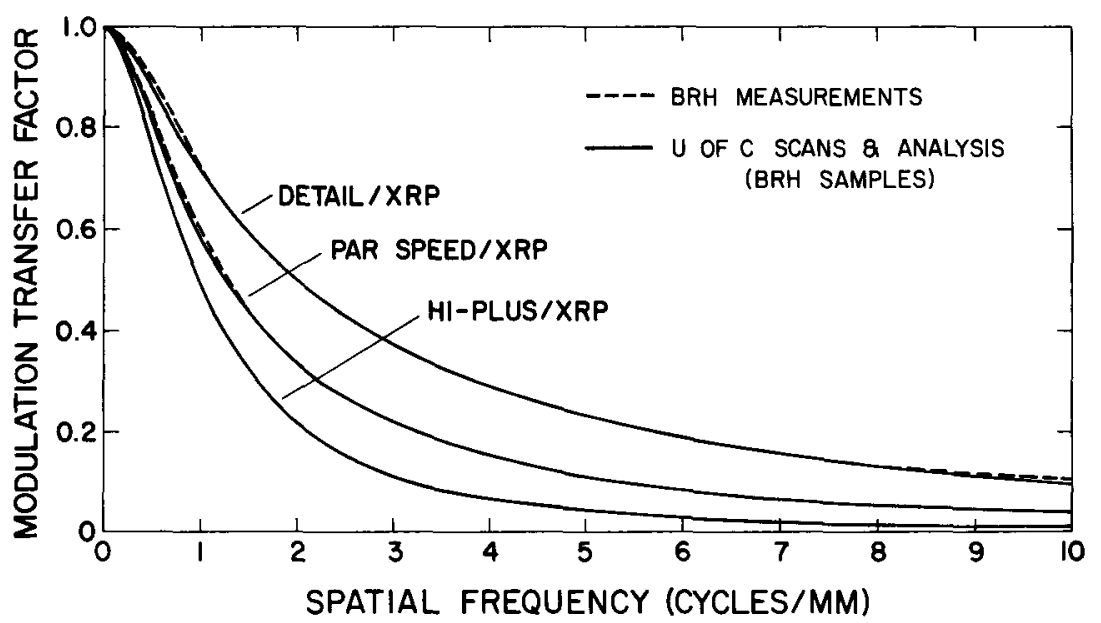

Fig 14. Comparison of MTF curves of three screens (using XRP film) as measured at CDRH (at that time the Bureau of Radiological Health) and the University of Chicago. ${ }^{35}$ ever, in part A the frequency spectrum of the noise power (NPS) adjoins that of the signal power, whereas in part B the NPS is shifted to higher frequencies. This shows that noise interferes with the interpretation of an image most when the noise and signal spectra are similar and that it interferes less otherwise. The spatial frequency distribution of the noise is important, not just the quantity of noise.

Note the immediate utility of MTF and NPS in comparing similar types of imaging systems. For example, in support of the claim of Fig 10 for a true factor-of-two gain in dose efficiency for the new phosphor screens, we carried out MTF and NPS measurements on both systems to show the equivalence of their heretofore subjective "image quality."
The MTF and NPS are quantitative measures of system output; however, they don't directly indicate the efficiency of a system or how well the system will perform on a given imaging task. For this we need to consider how much information the system provides to an observer to allow that observer to carry out a particular imaging task, and how much its counterpart, the ideally configured system, would similarly provide.

Our observer is the ideal Bayesian observer who operates within a maximum likelihood formalism to make decisions on, for example, whether or not the shadow of a lesion is present in a particular radiograph. ${ }^{39-42}$ Given two hypotheses $a$ and $b$, the ideal observer uses all available information to form the conditional probabilities $p(\underline{x} \mid a)$ and $p(\underline{x} \mid b)$ for a data set $\underline{x}$ and then uses
Fig 15. Comparison of NPS (Wiener spectra) of three screens (using XRP film) as measured at CDRH and the University of Chicago and given in terms of diffuse density. ${ }^{36}$

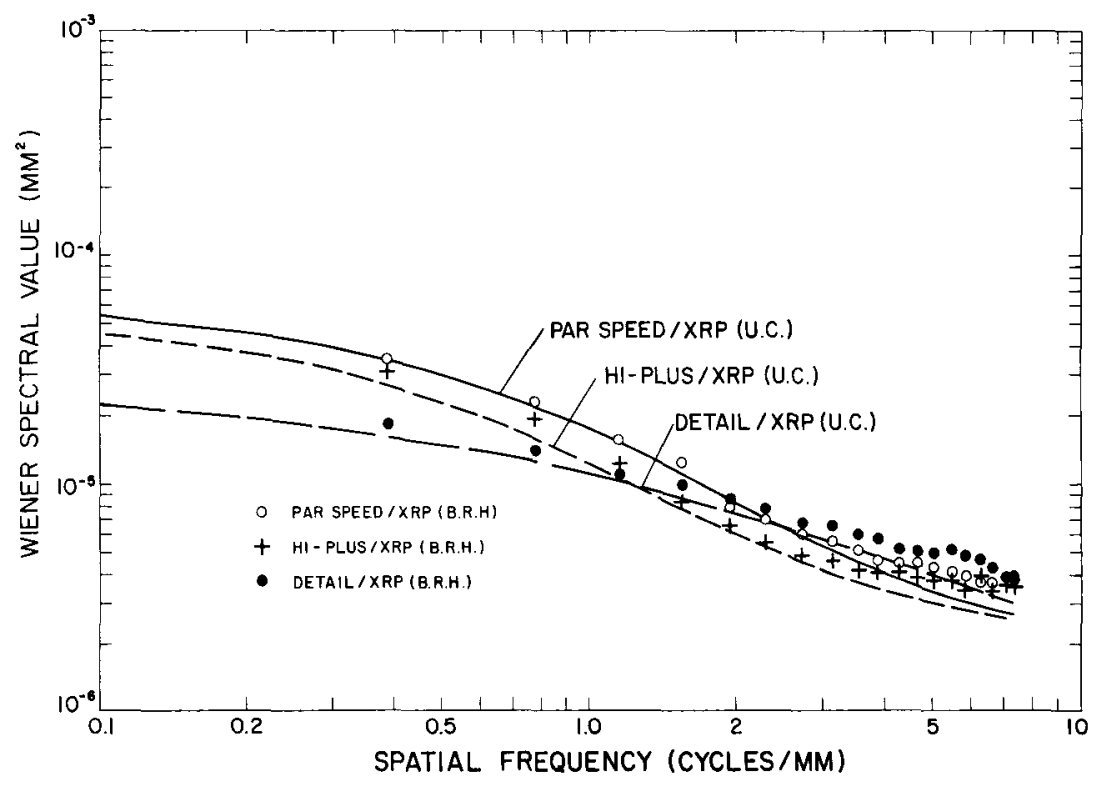



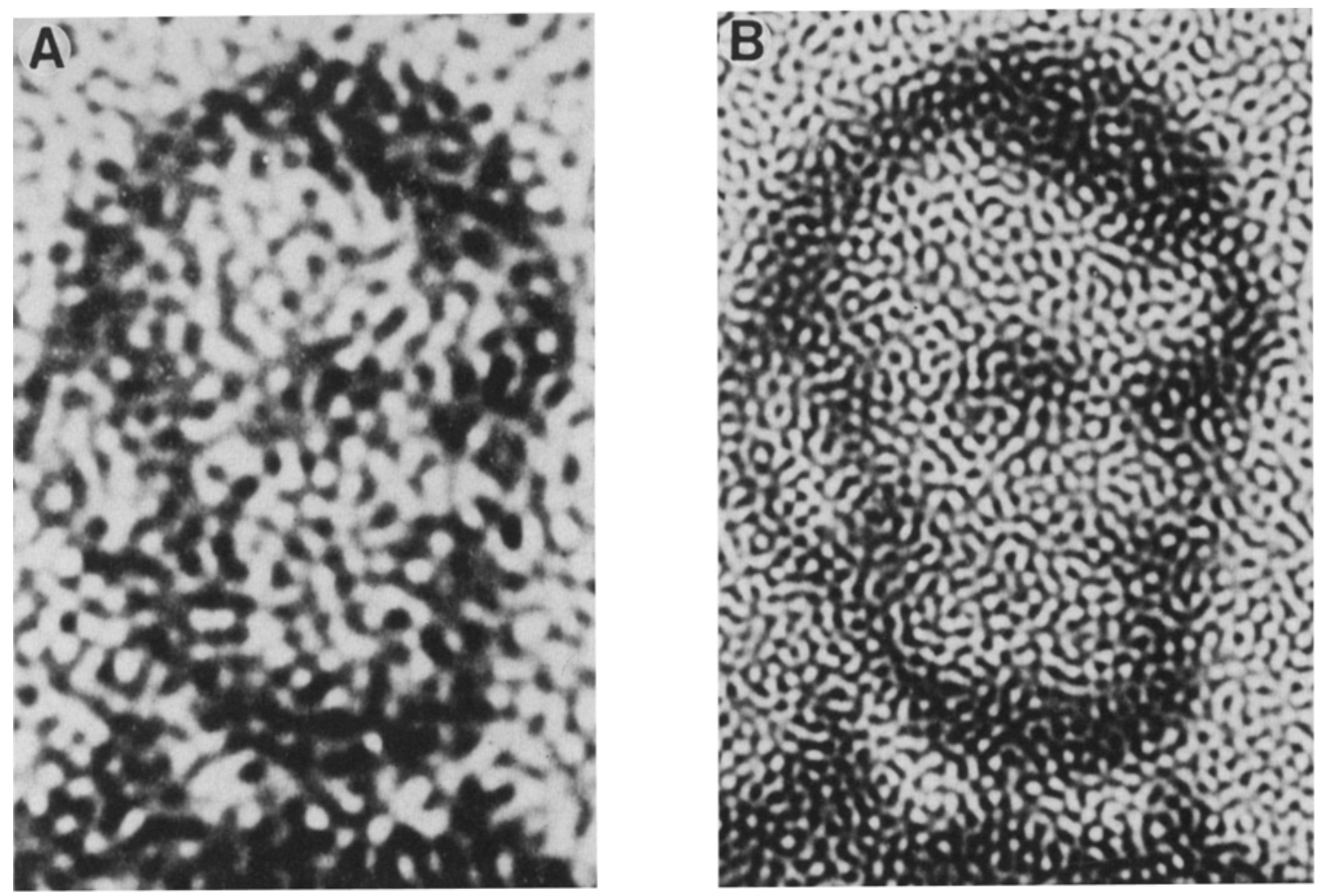

Fig 16. Low-band-pass filtered images of Lincoln with suppression of signal spatial frequencies above $f=10$ cycles. (A) Noise of uniform amplitude was added between $f$ and $4 f$ (ie, just adjacent to the portrait's spectrum): (B) noise was added in the spectral range $4 f$ to $7 f$. (Harmon LD. Julesz B: Science 180:1194-1197, 1973. 1973 by the AAAS.)

the likelihood ratio $\mathrm{L}=\mathrm{p}(\underline{\mathrm{x}} \mid \mathrm{a}) / \mathrm{p}(\underline{\mathrm{x}} \mid \mathrm{b})$ (or any monotonic function of $L$ ) as its decision variable in deciding which hypothesis the data supports. $L$ is essentially the "betting odds" for $a$ versus $b$ based on the results of the measurements $x$.

Intuitively, the form of the resulting decision variable seems appealing. The ideal observer weights most heavily the data for which the signal is largest and the noise is smallest. For the simple case of detection of a signal with independent, equal-variance Gaussian noise channels, this becomes template matching, with the ideal observer using as a template the shape of the signal (or matched filtering with the signal spectrum as a "filter" in the spatial frequency domain). The ideal observer is said to operate with a prewhitening matched filter because it accurately accounts for any spatial correlations in the noise, or lack of whiteness or uniformity of the noise power spectrum in the frequency domain. Sometimes a second, quasi-ideal observer is also of use, because it more closely follows the performance of a human observer. This is an observer that cannot take into account the correlations in the noise, but instead treats the data as if the noise were always white.

These ideas lead naturally to another important construct, that of the SNR. As already noted, it is intuitively appealing that increased signal strength should enhance performance on an imaging task and that increased noise should detract from it. Therefore, it is natural to use an SNR as a figure of merit for imaging performance. When it is also found that the performance of the ideal observer for simple imaging tasks is given in terms of an SNR, this inclination receives a solid scientific foundation. In what follows, some of the ramifications of these ideas will be explored, and SNRs for a number of imaging modalities will be presented. Usually squared SNRs will be given because from information theoretic considerations, it is actually the square of the SNR that is the fundamental figure of merit for imaging performance. ${ }^{20}$

Calculations of the SNR for a simple imaging task is illustrated in Fig 17. The task is detection 


\section{$\Delta s=\Delta n A\left(A=\pi r^{2}\right)$}

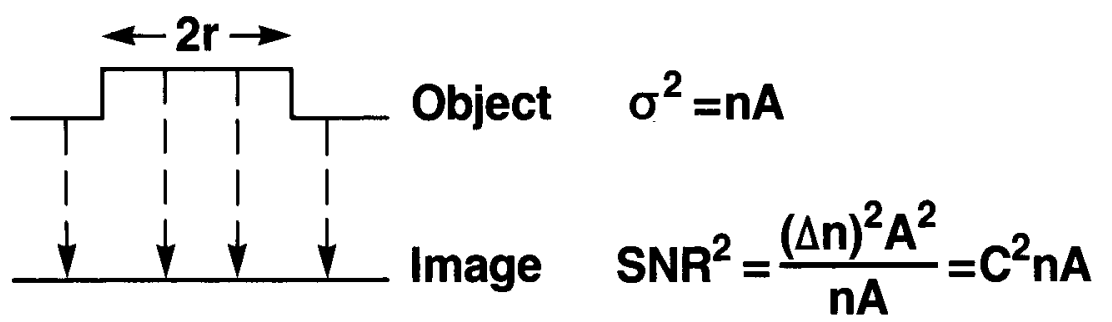

Fig 17. Simple classical (SNR) ${ }^{2}$ calculation for a low contrast "disk" shaped lesion of radius $r$ and area $A=\pi r^{2}$. of an area of increased activity (lesion) in a plane projection nuclear-medicine image. If the difference in detected activity between the lesion and the background is $\Delta \mathrm{n}$ counts $/ \mathrm{cm}^{2}$, and the mean detected activity at the detector is n counts $/ \mathrm{cm}^{2}$, and the lesion has a projected area on the detector of $\mathrm{A} \mathrm{cm}$, then the difference signal at the detector is $\Delta \mathrm{nA}$. Because this is specified to be a nuclear medicine system, we assume Poisson statistics for the random and independent arrival of photons at the detector, with the variance of any measurement equal to the number of photons detected, $\sigma^{2}=\mathrm{nA}$ (where we assume $\mathrm{n} \simeq \mathrm{n}+\Delta \mathrm{n}$; low-contrast case). Therefore we have the following result for the square of the SNR:

$$
\begin{aligned}
(\mathrm{SNR})^{2} & =(\Delta \mathrm{nA})^{2} /(\mathrm{nA}) \\
& =(\Delta \mathrm{n} / \mathrm{n})^{2} \mathrm{nA} \\
(\mathrm{SNR})^{2} & =\mathrm{C}^{2} \mathrm{~N}
\end{aligned}
$$

where $C$ is the detected contrast $\Delta \mathrm{n} / \mathrm{n}$ and $N=$ $\mathrm{nA}$ is the total number of counts received in the lesion shadow.

For reliable signal detection, $\mathrm{SNR}^{2}=\mathrm{C}^{2} \mathrm{~N}$ must be on the order of unity (from one to five). ${ }^{10}$ Thus, in $x$-ray imaging, in which typically $10^{4}$ counts $/ \mathrm{mm}^{2}$ are detected, a contrast of .01 or $1 \%$ can be detected for a $1-\mathrm{mm}^{2}$ lesion in the absence of scattered radiation. Similarly, in nuclear medicine for which about $10^{3}$ counts $/ \mathrm{cm}^{2}$ are detected, a contrast of a few percent can be detected for a $1-\mathrm{cm}^{2}$ lesion. Reconstruction imaging modalities, such as computed tomography, require a more careful analysis which will be touched on briefly below, because the noise in the reconstructed image is not white but is correlated by the reconstruction process.

Note how well this agrees with our earlier desire to assess each of the images of Fig 3 as worth a certain number of photons. The above equation for $(\mathrm{SNR})^{2}$ allows this idea to be quantified. The $(\mathrm{SNR})^{2}$ for detection of any given feature of the scene, such as the flower, is proportional to the square of the inherent image contrast and to the number of photons detected from that feature. Thus, if we measure SNR in the image (or, more properly, an ensemble of images) for an object of known contrast, we can calculate the number of photons that should have been required to produce the image. We refer to this as the noise equivalent quanta or NEQ. Further, if we know the actual number of photons (Q) available to the system, we can normalize NEQ to an efficiency called the detective quantum efficiency, $\mathrm{DQE}=\mathrm{NEQ} / \mathrm{Q}$.

This idea also helps to explain why the new phosphor screens mentioned earlier were able to reduce radiation exposure to the patient. In the above equation, $\mathrm{C}$ has not changed, but by cutting the exposure in half, one would expect $\mathrm{N}$ to also have been cut in half with a similar decrease in performance. Figure 18 indicates how this effect is counterbalanced by a factor-oftwo greater stopping power of the new screens. ${ }^{28}$ Previously a high percentage of the x-rays incident on the screens was not absorbed: "wasted" $\mathrm{x}$-rays contributed to patient dose but not to production of the radiograph. By increasing the efficiency of the screen, a lower exposure can now produce the same photon count at the detector and hence the same quality image.

These concepts can be extended to other imaging modalities, although, of course, the appropriate statistics and other details of the calculations are specific to the modality. In addition, they can be made more complete, just as was the case for resolution and noise measures, by calculating them as a function of spatial frequency rather than giving a single value. For example, NEQ can be shown to assume the following form: 
Fig 18. Top to bottom: $X-$ ray spectra for radiation: 1 , incident upon screens; 2 , exiting from a pair of traditional calcium tungstate screens (TF-2); 3, exiting from an early pair of rare earth screens (Alpha 8); 4, exiting from a different pair of rare earth screens (Lanex). Note greater absorption in rare earth screens. ${ }^{28}$

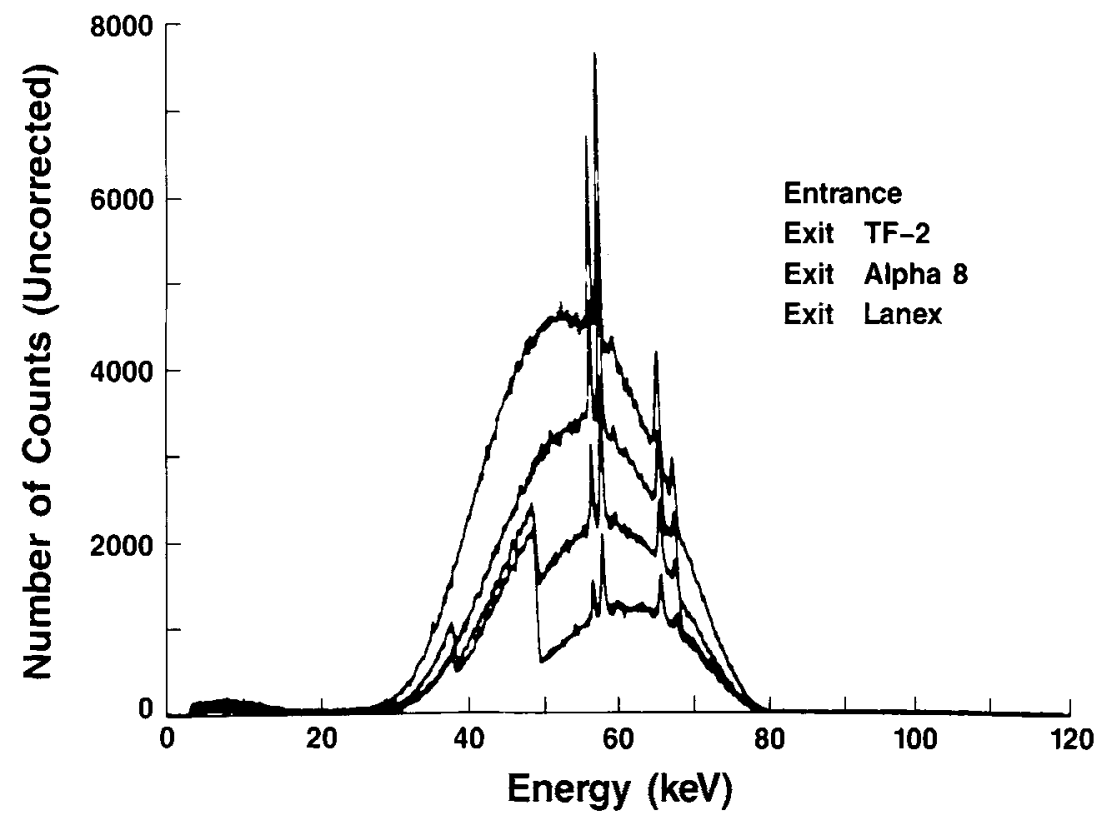

$$
\operatorname{NEQ}(f)=\operatorname{MTF}(f)^{2} / N P S(f)
$$

and for $(\mathrm{SNR})^{2}$ we have:

$$
(\mathrm{SNR})^{2}(\mathrm{f})=\mathrm{C}^{2}(\mathrm{f}) \mathrm{NEQ}(\mathrm{f})
$$

so that, in general,

$$
(\mathrm{SNR})^{2}=\int \mathrm{C}^{2}(\mathrm{f}) \mathrm{NEQ}(\mathrm{f}) \mathrm{df}
$$

An example of NEQ(f) is given in Fig 19, which shows NEQ(f) for two CT systems. ${ }^{43}$ Using this approach, we were able to show that even these early CT systems were reasonably efficient, and that proposed order-of-magnitude gains in dose efficiency from special processing algorithms were a fiction. ${ }^{13}$

Wagner and Brown ${ }^{20}$ give SNRs for many different imaging systems. The calculations for the more complicated system geometries are too complex to reproduce fully here; however, we will briefly discuss the approach for reconstruction from projections, as in CT and PET. ${ }^{44}$ The geometry for PET imaging is indicated in Fig 20 for both the spatial and spatial-frequency domains. The uniform Poisson noise processes in the projections add up to a $1 / f$ dependence for the noise in polar coordinates in frequency space-as evidenced by the relatively tight packing of the data near the origin shown in the figure (When the noise is filtered through the reconstruc-

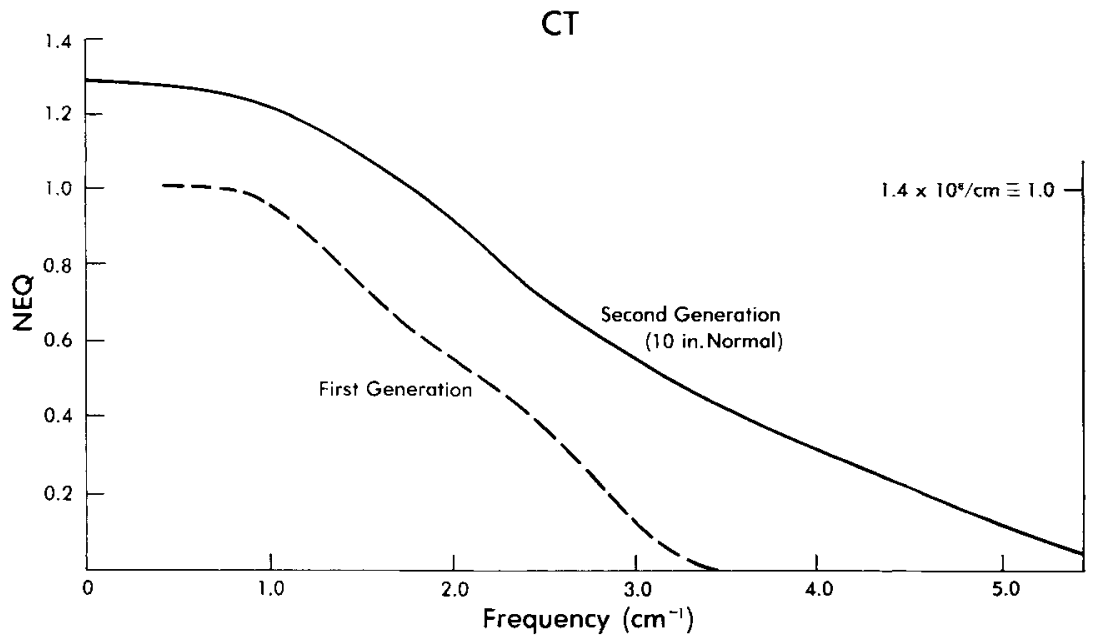

Fig 19. NEO(f) spectra for a first- and second-generation CT system (EMI) operating at typical technique factors. The radiation dose for both systems was approximately equal, implying that the second-generation machine had a small but significent edge in dose efficiency over the other system. ${ }^{43}$ 


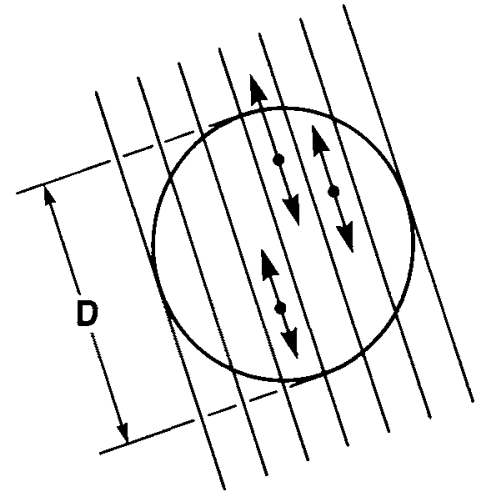

Spatial Domain

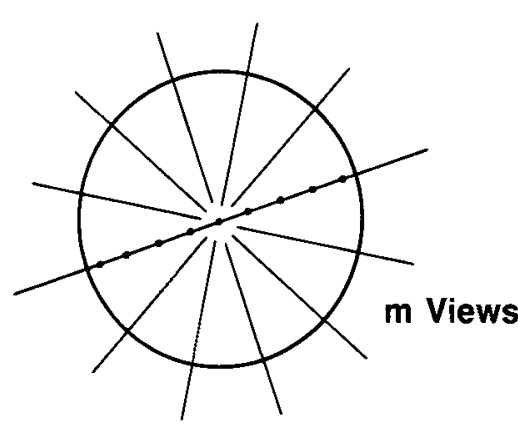

Frequency Domain
Fig 20. At left, spatial domain geometry for ray projection tomography, showing strip integrals land positron annihilation events, for the case of PET) for a single view. At right, frequency domain geometry. with the "slice" with tick marks corresponding to the given view, and other rays indicating other projection angles. ${ }^{44}$ tion ramp filter, ie, multiplied by two powers of $f$, because the noise is second order in the data, the familiar result of NPS being proportional to $f$ emerges.) The same transformation is performed for the signal, and one obtains (SNR) ${ }^{2}$ as a function of spatial frequency. This is then integrated over frequency channels, yielding for the case of detection of a Gaussian lesion:

$$
(\mathrm{SNR})_{\mathrm{PET}}^{2}=\mathrm{C}^{2}(\mathrm{a} / 2)^{2}\left(\rho \mathrm{m} / \mathrm{Da}^{1 / 2}\right)
$$

where $a$ is the effective area of the lesion, $m$ is the number of views used in making the reconstruction, $D$ is the overall diameter of the cross section of patient anatomy, $\rho$ is the mean count density per unit area, and $C=\Delta \rho / \rho$.

This expression is of intrinsic interest for its description of the dependence of (SNR) ${ }^{2}$ on the variables on the right side of the equation. It is also valuable in allowing for comparisons with other imaging modalities. It may be compared with the result for exact time-of-flight PET (TOFPET), for example, where the position of the radioactive decay is known exactly and for which

$$
(\mathrm{SNR})_{\mathrm{TOFPET}}^{2}=\mathrm{C}^{2}(\mathrm{a} / 2)^{2}(\rho \mathrm{m} / \mathrm{a}) .
$$

(Note that this is simply $\mathrm{C}^{2} \mathrm{~N}$ because a $\rho$ is the number of counts per view and $m$ is the number of views. The extra factor of two enters because we are using a Gaussian and not a disk shaped lesion. The more complicated expression for PET resulted from the reconstruction process.) Therefore, we have for their ratio,

$$
\frac{(\mathrm{SNR})_{\mathrm{PET}}^{2}}{(\mathrm{SNR})_{\mathrm{TOFPT}}^{2}}=\frac{\mathrm{a}^{1 / 2}}{\mathrm{D}} .
$$

Thus, in PET imaging, failure to know the exact location of the radioactive decay within the projected line extorts a penalty, the magnitude of which is the ratio of the effective linear dimension of the lesion being detected to that of the total region under consideration. This is the so-called multiplex penalty for imaging with line integrals as illustrated in Fig 21.

\section{Display Stage}

The final mission of any imaging modality is to efficiently couple the information obtained by its detection stage to the eye-brain system of a human observer. To accomplish this goal requires both due regard to the ergonomics common to all machine/human interactions and attention to the special psychophysical aspects of the human as image interpreter. As the data

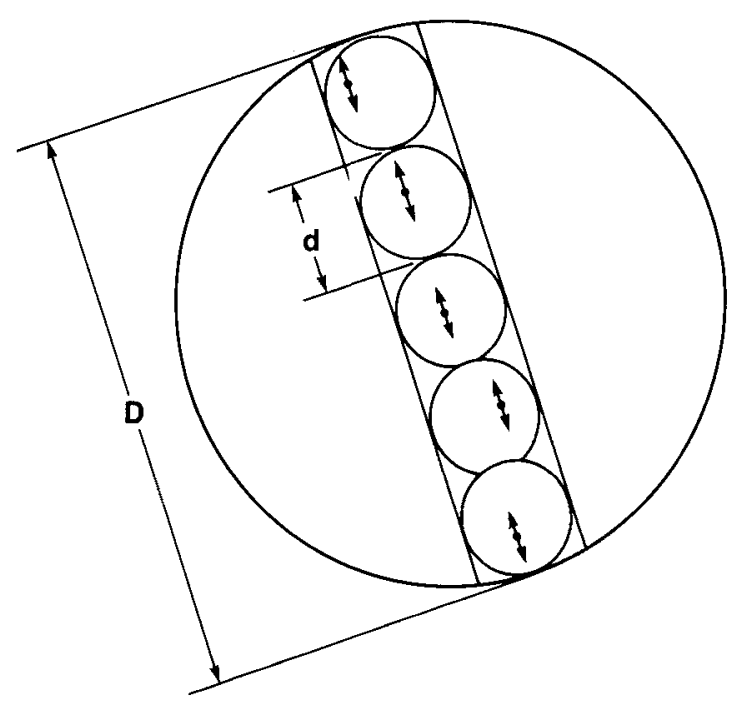

Fig 21. Origin of the multiplex penalty for planar reconstruction imaging, with the noise from D/d lesion-sized regions corrupting the strip integrals for each projection. 4 
available for display from modern systems has increased, the job of effectively presenting it has become more and more complex, and many new and innovative display technologies have been developed. ${ }^{31}$

Whatever method of display is used for a system, the performance of real observers on that system is amenable to the same quantitative assessment as for the system's detection stage. ${ }^{45-48}$ In this case, the real observer's performance is assessed on the same scale as that of an ideal observer-one that makes perfect use of all of the information presented to it by the detection stage of the system. A comparison of the two then shows the efficiency of real observers on the system and the degree to which their performance could possibly be improved.

One method of measuring human-observer performance is by generating the so-called "contrast-detail" diagram, in which the contrast required to detect a disklike lesion with some specified confidence or threshold is presented as a function of the lesion diameter. ${ }^{49}$ It is unlikely that human observers can maintain a specified confidence level, and the results are sensitive to this. ${ }^{49}$ There has been a trend toward implementing this scheme using a machine observer, particularly for evaluating image-reconstruction techniques. ${ }^{50}$

The problem of the observer's threshold is finessed by two techniques that have greatly matured in the last decade. The first is the two-alternative forced-choice (2AFC) experiment, in which the observer specifies which of two test images is the one containing a lesion, and the percentage of correct responses is recorded as a function of some imaging parameter such as signal size, contrast, or noise. The second technique is the method of mapping out the receiver operating characteristic (ROC) curve, a plot of true positive fraction (hits) versus false positive fraction (false alarms) as a function of the strictness or laxity of the observer in calling a lesion present. ${ }^{51}$ The ROC curve is usually generated in a single pass by having the observer specify the confidence in the call. The ROC results are related to the $2 A F C$ results by the area under the ROC curve. This measure is equal to both the average hit rate, averaged over all false-positive rates, and the percent correct score in a $2 \mathrm{AFC}$ experiment, using the same test images when the test comprises equal numbers of positives and negatives.

It has been found, using 2AFC experiments, that real observer performance can come very close to ideal observer performance, except in the cases of unprocessed (eg, unwindowed) lowcontrast images and images obtained through projection-reconstruction methods, for which there is a small but significant discrepancy..$^{20,45-48}$ Equivalently, given sufficient image contrast, the real observer performance approaches that of the previously defined quasi-ideal observer, which ignores noise correlations.

Results for a typical observer performance experiment are shown in Fig 22, which indicates how actual and ideal observer performances may be compared. ${ }^{52}$ The reports of Burgess et al ${ }^{45-48}$ and Myers et al ${ }^{53,54}$ are a good introduction to work in this field. More general information on such techniques as receiver operating characteristic analysis can be found, for example, in texts such as that by Swets and Pickett. ${ }^{55}$

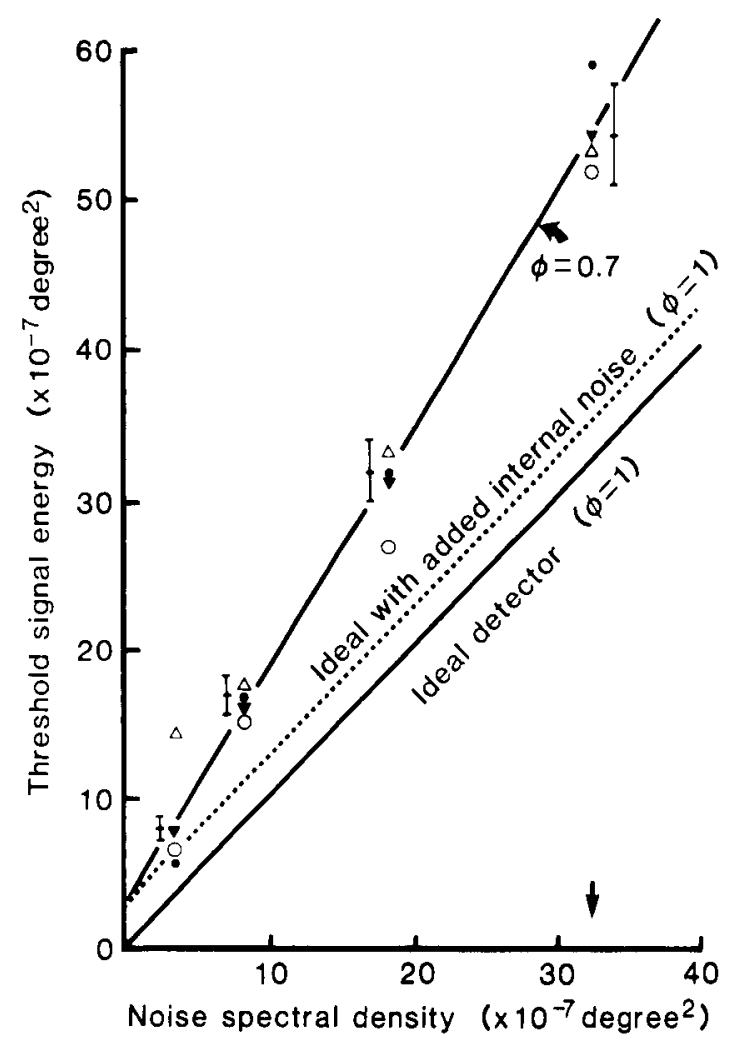

Fig 22. Signal energy needed for $76 \%$ correct decision in a two-alternative forced-choice experiment. Results for the ideal detector with and without an internal source of noise are shown. ${ }^{52}$ 


\section{CONCLUSION}

The menu of medical diagnostic imaging systems offers great variety, from the staple fare of plain film radiography to exotic innovations such as SQUID. However, all of these systems share common ingredients. Each investigates some physical characteristic or parameter of the patient's condition, and that parameter is described by its own unique physical and statistical at- tributes. Each system makes use of one or more forms of radiation and a more-or-less complicated chain of system components to interrogate the patient's body and to detect information about the parameter of interest. Each system is designed to present the detected information to a human observer for final analysis. Finally, all of the systems are amenable to evaluation of their performance in terms of the general approach outlined here.

\section{REFERENCES}

1. Ter-Pogossian MM: The Physical Aspects of Diagnostic Radiology. New York, NY, Harper \& Row, 1967

2. Kelley JP: History of the development of diagnostic x-ray systems, in Wright DJ (ed): Physics of Diagnostic Radiology. USDHEW Publication No FDA 74-8006, 1973, pp 2-50

3. Sorenson J, Phelps M: Physics in Nuclear Medicine. New York, NY, Grune \& Stratton, 1980

4. Wells PNT: Biomedical Ultrasonics. San Diego, CA, Academic, 1977

5. Newton TH, Potts DG (eds): Radiology of the Skull and Brain 5: Technical Aspects of Computed Tomography. St Louis, MO, Mosby, 1981

6. Walker MD (ed): Research issues in positron emission tomography. Annals of Neurology 15:S1-S204, 1984 (suppl)

7. Partain CL, Price RR, Patton JA, et al (eds): Magnetic Resonance Imaging. Philadelphia, PA, Saunders, 1988

8. Kruger RA, Riederer SJ: Basic Concepts of Digital Subtraction Angiography. Boston, MA, Hall, 1984

9. Alvarez RE: Limitations on biomagnetic imaging, in Schneider RH, Dwyer SJ III (eds): Medical Imaging II: Image Formation, Detection, Processing, and Interpretation. Proc SPIE 914:2-9, 1988

10. Rose A: Vision: Human and Electronic. New York, NY, Plenum, 1974

11. Shaw R: Evaluating the efficiency of imaging processes. Rep Prog Phys 41:1103-11 55, 1978

12. Dainty JC, Shaw R: Image Science, New York, NY, Academic, 1974

13. Wagner RF, Brown DG, Pastel MS: Application of information theory to the assessment of computed tomography. Med Phys 6:83-94, 1979

14. Wagner RF, Barnes GT, Askins BS: Effect of reduced scatter on radiographic information content and patient exposure: A quantitative demonstration. Med Phys 7:13-18, 1980

15. Alvarez RE: Extraction of energy dependent information in radiography. Doctoral thesis, Stanford University, Palo Alto, CA, 1976; see also Alvarez RE, Macovski A: Energy-selective reconstructions in $\mathrm{x}$-ray computerized tomography. Phys Med Biol 21:733-744, 1976

16. Alvarez RE, Marshall WH, Lewis R: Tissue characterization using energy-selective computer tomography, in Gray JE, Haus AG, Properzio WS, et al (eds): Application of Optical Instrumentation in Medicine IX. Proc SPIE 273:301307,1981
17. Sones RA, Tesic MM, Barnes GT: Dual-energy chest radiography. Physics Today S44-S45, 1987 (suppl)

18. Mazziotta JC, Phelps ME: Human sensory stimulation and deprivation: Positron emission tomographic results and strategies. In Walker MD (ed): Research Issues in Positron Emission Tomography. Ann Neurol 15:S50-S60, 1984 (suppl)

19. Kressel HY (ed): Magnetic Resonance Annual: 1988. New York, NY, Raven, 1988

20. Wagner RF, Brown DG: Unified SNR analysis of medical imaging systems. Phys Med Biol 30:489-518, 1985

21. Macovski A: Medical Imaging Systems. Englewood Cliffs, NJ, Prentice-Hall, 1983

22. Barrett HH, Swindell W: Radiological Imaging: The Theory of Image Formation, Detection, and Processing. New York, NY, Academic, 1981

23. Committee on the Biological Effects of Ionizing Radiations: The Effects on Populations of Exposure to Low Levels of Ionizing Radiation. Washington, DC, National Academy of Sciences, 1980

24. American Institute of Ultrasound in Medicine Bioeffects Committee: Bioeffects considerations for the safety of diagnostic ultrasound. Ultrasound Med 7, 1988 (suppl)

25. Wagner RF, Weaver KE: Prospects for $x$-ray exposure reduction using rare-earth intensifying screens. Radiology 118:183-188, 1976

26. Wagner RF: Fast Fourier digital quantum mottle analysis with application to rare-earth intensifying screen systems. Med Phys 4:157-162, 1977

27. Larsen LE, Jacobi JH (eds): Medical Applications of Microwave Imaging. New York, NY, Institute of Electrical and Electronics Engineers, 1986

28. Wagner RF, Jennings RJ: The bottom line in radiologic dose reduction. Proc SPIE 206:60-66, 1979

29. Muntz EP, Jafroudi H, Jennings R, Bernstein H: An approach to specifying a minimum dose system for mammography using multiparameter optimization techniques. Med Phys 12:5-12, 1985

30. Gagne RM: Geometrical aspects of computed tomography: Sensitivity profile and exposure profile. Med Phys 16:29-37, 1989

31. Medical imaging (special feature). Computers in Physics 2:16-49, 1988

32. Joseph PM: Artifacts in computed tomography, in Newton TH, Potts DG (eds): Radiology of the Skull and 
Brain 5: Technical Aspects of Computed Tomography. St Louis, MO, C.V. Mosby, 1981 pp 3596-3992

33. International Commission on Radiation Units and Measurements: Modulation transfer function of screen-film systems. ICRU Report 41, 1986

34. O'Neil EL: Introduction to Statistical Optics. Reading, MA, Addison-Wesley, 1963

35. Doi K, Holje G, Loo L-N, Chan H-P, Sandrik JM, Jennings RJ, Wagner RF: MTF's and Wiener spectra of radiographic screen-film systems. Washington, DC, US Dept HHS, Publ. FDA 82-8187, US GPO, 1982

36. Sandrik JM, Wagner RF, Hanson KM: Radiographic screen-film noise power spectrum: Calibration and intercomparison. Appl Opt 21:3597-3601, 1982

37. Sandrik JM, Wagner RF: Absolute measures of physical image quality: Measurement and application to radiographic magnification. Med Phys 9:540-549, 1982

38. Harmon LD, Julesz B: Masking in visual recognition: effects of two-dimensional filtered noise. Science 180:1194 1197,1973

39. Van Trees HL: Detection, Estimation, and Modulation Theory. New York, NY, Wiley, 1968

40. Whalen AD: Detection of Signals in Noise. Orlando, FL, Academic, 1971

41. Fukunaga K: Introduction to Statistical Pattern Recognition. Orlando, FL, Academic, 1972

42. Green DM, Swets JA: Signal Detection Theory and Psychophysics. Huntington, NY, R.E. Krieger, 1974

43. Wagner RF, Brown DG: Overview of a unified SNR analysis of medical imaging systems. IEEE Transactions on Medical Imaging MI-1:210-214, 1982

44. Wagner RF, Brown DG: From the Rose model to diffraction tomography: Statistics of low contrast images, in Morris GM (ed): Statistical Optics. Proc SPIE 976:101-108, 1988
45. Burgess A, Ghandeharian H: Visual signal detection. I. Ability to use phase information. J Opt Soc Am A1:900-905, 1984

46. Burgess AE, Ghandeharian H: Visual signal detection. II. Signal-location identification. J Opt Soc Am Al:906-910, 1984

47. Burgess A: Visual signal detection. III. On Bayesian use of prior knowledge and cross correlation. J Opt Soc Am A2:1498-1507, 1985

48. Burgess AE, Colborne B: Visual signal detection. IV. Observer inconsistency. J Opt Soc Am A5:61 7-627, 1988

49. Wagner RF, Metz CE, Brown DG: Signal detection theory and medical image assessment, in Doi K, Lanzi L, Lin P-JP (eds): Recent Developments in Digital Imaging. New York, NY, American Institute of Physics, 1985, pp 39-59

50. Hanson KM: Method to evaluate image-recovery algorithms based on task performance. Proc SPIE 914:336343, 1988

51. Metz CE: ROC Methodology in Radiologic Imaging. Invest Radiol 21:720, 1986

52. Burgess AE, Wagner RF, Jennings RJ, Barlow HB: Efficiency of human visual discrimination. Science 214:9394,1981

53. Myers KJ, Barrett HH, Borgstrom MC, Patton DD, Seeley GW: Effect of noise correlation on detectability of disk signals in medical imaging. J Opt Soc Am A2:1752-1759, 1985

54. Myers KJ, Barrett HH: Addition of a channel mechanism to the ideal-observer model. J Opt Soc Am A4:24472457,1987

55. Swets JA, Pickett RM: Evaluation of Diagnostic Systems: Methods from Signal Detection Theory. New York, NY, Academic, 1982 